Mathematical Modelling and Analysis

Volume 6 Number 2, 2001, pages 192-198

(C) 2001 Technica

\title{
ANALYSIS OF GENERALIZED MULTISTEP ADAM'S METHODS BY DEGENERATE MATRIX METHOD FOR ORDINARY DIFFERENTIAL EQUATIONS
}

\author{
T. CĪRULIS, O. LIETUVIETIS \\ Institute of Mathematics of Latvian Academy of Sciences \\ and University of Latvia \\ Akadēmijas laukums 1, Rīga LV-1524, Latvia \\ E-mail: cirulis@lanet.lv ojarsl@lanet.lv
}

Received October 1, 2001; revised October 24, 2001

\begin{abstract}
Adam's methods in the multistep mode are considered by means of general schemes of the degenerate matrix method. The stability function for these methods is computed by the residue theory on the complex plane. Performance of uniformly and non-uniformly distributed nodes in the standardized interval is compared.
\end{abstract}

\section{INTRODUCTION}

The degenerate matrix method (DM method) is a special computing scheme for numerical solutions of initial values problems of ODE. It is analyzed in the literature, for example, in $[1,2]$. DM methods are based on the use of matrices of two types: matrices for derivatives $\boldsymbol{\Delta}_{N}$ which are always degenerate and/or their pseudo-inverses $\mathbf{B}_{N}$ at the fixed system of $N$ nodes on the standardized interval. DM-method in the one step mode was analyzed in [3]. Now we will investigate an application of ones in the multistep mode. Some of DM methods in the multistep mode which use only of last row of $\boldsymbol{\Delta}_{N}$ or the last row of $\mathbf{B}_{N}$ were also analyzed in [4]. Now we will investigate Adam's methods which can be very simply constructed using two rows of pseudo-inverse matrices $\mathbf{B}_{N}$. Generally these Adam's methods depend on an arbitrary constant and have non-uniformly distributed nodes in the standard interval $[-1,1]$. An application of DM-methods in the mode of Adam's schemes gives a simple possibility both 
to form these computing schemes and to analyze them. A stability of methods is investigated separately using the function theory of complex variables as in [4]. There are also considered different examples, where the stability domains for different Adam's methods in the case of uniformly distributed nodes with ones for non-uniformly distributed nodes are compared.

\section{FORMULATION OF PROBLEM AND COMPUTING SCHEMES}

The equation $y^{\prime}=f(t, y)$ with the initial value $y\left(t_{0}\right)=y_{0}$, where $y, y_{0}, f \in \mathbb{R}^{n}$, must be solved in the interval $\left[t_{0}, T\right]$. We will use in the multistep mode a step by step procedure based on applications of DM schemes. To this end it is convenient each subinterval $\left[t_{j}, t_{j}+H\right]$ to reduce to the standard interval $[-1,1]$ by means of the linear substitution

$$
t=t_{j}+0.5 H(x+1) .
$$

Here $H$ which can be also depended on $j$ is the length of the mold in the $j$-th step, but $(2.1)$ maps interval $[-1,1]$ into $\left[t_{j}, t_{j}+H\right]$. Therefore our main attention will be devoted to solving the problem

$$
y^{\prime}=\frac{H}{2} F(x, y),\left.\quad y\right|_{x=-1}=\alpha
$$

in the interval $x \in[-1,1]$, but $F(x, y)=f\left(t_{j}+0.5(H+1), y\right)$;

$\alpha=y\left(t_{j}\right)$. Let

$$
-1 \leq x_{0}<x_{1}<\ldots,<x_{N}<x_{N+1}=1
$$

be nodes of the standardized interval and

$$
p_{N+2}(x)=(x-1) q_{N+1}(x), \quad q_{N+1}(x)=\prod_{k=0}^{N}\left(x-x_{k}\right)
$$

are polynomials. Let $\boldsymbol{\Delta}_{N+2}$ be the matrix for derivatives according to nodes (2.3) and $\mathbf{B}_{N+2}$ - their pseudo-inverse defined by the equality

$$
\mathbf{B}_{N+2} \boldsymbol{\Delta}_{N+2}=\mathbf{E}_{N+2}-\mathbf{I}_{N+2},
$$

where $\mathbf{E}_{N+2}$ is the identity matrix, but all elements of $\mathbf{I}_{N+2}$ are zeroes except the first column consisting of 1's. Elements $b_{i k}$ of the matrix $\mathbf{B}_{N+2}$ are

$$
b_{i k}=\frac{1}{p_{N+2}^{\prime}\left(x_{k}\right)}\left[\int_{x_{0}}^{x_{i}} \frac{p_{N+2}(\tau) d \tau}{\tau-x_{k}}+c_{k}\right] ; i, k=0,1, \ldots, N+1
$$


where $c_{k}$ are arbitrary constants [3]. Applying two rows of $\mathbf{B}_{N+2}$ with the numbers $i=N+1$ and $i=m$ to the problem (2.2) leads to the equations

$$
\begin{aligned}
& y_{N+1}=y_{0}+\frac{H}{2} \sum_{k=0}^{N+1} b_{N+1, k} F\left(x_{k}, y_{k}\right), \\
& y_{m}=y_{0}+\frac{H}{2} \sum_{k=0}^{N+1} b_{m, k} F\left(x_{k}, y_{k}\right),
\end{aligned}
$$

and,after the subtracting, we obtain the scheme:

$$
\begin{gathered}
y_{N+1}=y_{m}+\frac{H}{2} \sum_{k=0}^{N+1} a_{k} F\left(x_{k}, y_{k}\right), \\
a_{k}:=b_{N+1, k}-b_{m, k}=\frac{1}{p_{N+2}^{\prime}\left(x_{k}\right)}\left[\int_{x_{m}}^{1} \frac{p_{N+2}(\tau) d \tau}{\tau-x_{k}}+c\right],
\end{gathered}
$$

where $m$ is one of fixed numbers $0,1, \ldots, N$ and $c=c_{N+1}-c_{m}$ is an arbitrary constant. The choice $m=0$ in $(2.7),(2.8)$ leads to the scheme analyzed in [4], but one with $m=N$ - to the generalized Adam's schemes which depend also on one arbitrary constant $c$ :

$$
\begin{array}{r}
y_{N+1}=y_{N}+\frac{H}{2} \sum_{k=0}^{N+1} b_{k} F\left(x_{k}, y_{k}\right), \\
b_{k}:=b_{N+1, k}-b_{N, k}=\frac{1}{p_{N+2}^{\prime}\left(x_{k}\right)}\left[\int_{x_{N}}^{1} \frac{p_{N+2}(\tau) d \tau}{\tau-x_{k}}+c\right] .
\end{array}
$$

Choosing $c$ according to the equality $b_{N+1}=0$ we obtain the following explicit Adam's scheme:

$$
\begin{aligned}
& y_{N+1}=y_{N}+\frac{H}{2} \sum_{k=0}^{N} g_{k} F\left(x_{k}, y_{k}\right), \\
& g_{k}=\frac{1}{q_{N+1}^{\prime}\left(x_{k}\right)} \int_{x_{N}}^{1} \frac{q_{N+1}(\tau) d \tau}{\tau-x_{k}}, q_{N+1}(x)=\prod_{k=0}^{N}\left(x-x_{k}\right) .
\end{aligned}
$$

Another choices of $c$ lead to implicit schemes.

\section{STABILITY FUNCTION FOR METHODS}

We will define the first stability function $R_{1}(z):=y_{N+1} / y_{0}$ for the computing scheme (2.7), (2.8) of Dalquist test problem

$$
y^{\prime}=z y,\left.\quad y\right|_{x=x_{0}}=y_{0}, \quad z=\frac{H \lambda}{2} \in \mathbb{C},
$$


if $y_{N+1}$ is computed according to scheme (2.7), (2.8) and $y_{0}, y_{1}, \ldots, y_{N}$ are known as starting values. Applying the computations by the residues theory of complex functions leads to the following result.

The stability function $R_{1}(z)$ for the computing scheme (2.7), (2.8) has a representation:

$$
\begin{aligned}
& R_{1}(z)=\exp \left[z\left(1-x_{0}\right)\right]-\Psi(z) ; \Psi(z)=\frac{\exp \left(-z x_{0}\right)}{1-z a_{N+1}} \phi(z), \\
& \phi(z)=\frac{z^{N+3}}{(N+2) !} \sum_{k=0}^{+\infty} \frac{d_{k} z^{k}}{(N+3)_{k}}-\frac{c z^{N+2}}{(N+1) !} \sum_{k=0}^{+\infty} \frac{s_{k} z^{k}}{(N+2)_{k}},
\end{aligned}
$$

where

$$
d_{k}=\sum_{j=0}^{k} \gamma_{j} s_{k-j} ; \quad \gamma_{j}=\int_{x_{m}}^{1} \tau^{j} p_{N+2}(\tau) d \tau,
$$

and $s_{j}$ are coefficients of the Laurent series

$$
\frac{1}{p_{N+2}(\tau)}=\tau^{-N-1} \sum_{j=0}^{+\infty} s_{j} \tau^{-j}, \quad|\tau|>1
$$

The series in (3.2) are convergent in all complex $z$ plane. In addition, they present the asymptotic series for $R_{1}(z)$ when $z \rightarrow 0$.

The proof of this representation (3.2) is similar to one in [4]. Using for the test problem (3.1) the computing scheme $(2.7),(2.8)$ we obtain

$$
\left(1-a_{N+1}\right) R_{1}(z)=\exp \left[z\left(x_{m}-x_{0}\right)\right]+z \exp \left(-z x_{0}\right) \sum_{k=0}^{N} a_{k} \exp \left(z x_{k}\right),
$$

where

$$
a_{k}=\frac{Q_{N+1}\left(x_{k}\right)}{p_{N+2}^{\prime}\left(x_{k}\right)}, Q_{N+1}(s)=\int_{x_{m}}^{1} \frac{p_{N+2}(\tau)-p_{N+2}(s)}{\tau-s} d \tau+c .
$$

Using for $a_{k}$ the representations (3.6) and computing the sum in (3.5) leads to (3.2). The proof of (3.2) is completed.

For Adam's scheme (2.9) and (2.10) we have the representation of the stability function $R_{1}(z)$ also in the form (3.2), but now the coefficients $\gamma_{j}$ are determinated by

$$
\gamma_{j}=\int_{x_{N}}^{1} \tau^{j} p_{N+2}(\tau) d \tau
$$

instead of (3.3). 
Analogously we have representations for the second, third and further stability functions of generalized Adam's methods in the following form $R_{2}(z):=$ $y_{N+2} / y_{0}, R_{3}(z):=y_{N+3} / y_{0}$. For example, in case $x_{0}=-1, x_{m}=x_{N}$, for schemes (2.9), (2.10) we obtain the following functions

$$
\begin{array}{r}
R_{2}(z)=\exp (h z) R_{1}(z)-g_{1}(z) \Psi(z), \quad g_{1}(z)=\frac{1+z b_{N}}{1-z b_{N+1}} ; \\
R_{3}(z)=\exp (h z) R_{2}(z)-g_{2}(z) \Psi(z), \\
g_{2}(z)=\left(\frac{1+z b_{N}}{1-z b_{N+1}}\right)^{2}+\frac{z b_{N-1} \exp \left[z\left(x_{N-1}-x_{N}+h\right)\right]}{1-z b_{N+1}} ; \\
R_{4}(z)=\exp (h z) R_{3}(z)-g_{3}(z) \Psi(z), \\
g_{3}(z)=\left(\frac{1+z b_{N}}{1-z b_{N+1}}\right)^{3}+\frac{z b_{N-1}\left(1+z b_{N}\right)}{\left(1-z b_{N+1}\right)^{2}} \exp \left[z\left(x_{N-1}-x_{N}+h\right)\right]+ \\
+\frac{z b_{N-2}}{1-z b_{N+1}} \exp \left[z\left(x_{N-2}-x_{N}+2 h\right)\right] .
\end{array}
$$

These results show that the first stability function $R_{1}(z)$ is an asymptotic expansion of further stability functions $R_{m}(z):=y_{N+m} / y_{0}, m=2,3, \ldots$ when $z \rightarrow 0$. Therefore, the inequality $\left|R_{1}(z)\right|<1$ gives the stability domain of Adam's methods correctly only for small enough $|z|$ or for small enough length $H$ of the mold.

\section{NUMERICAL EXPERIMENTS}

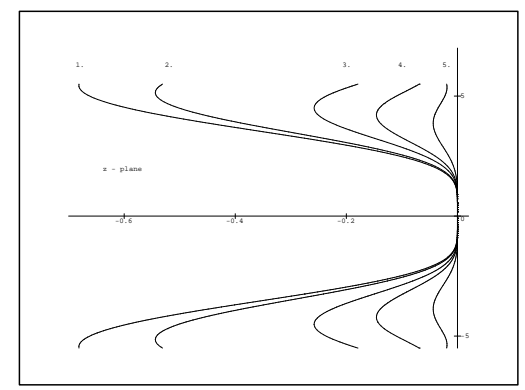

Figure 1. The borders of instability regions for three-step Adam's methods.

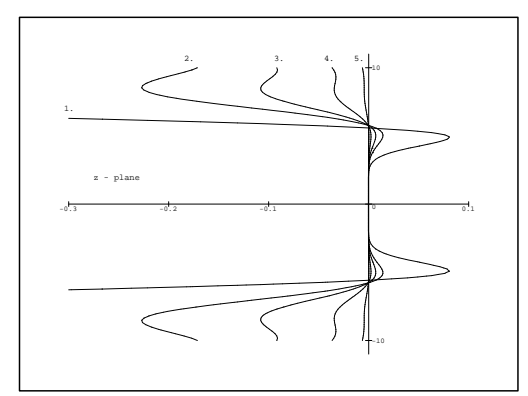

Figure 2. The borders of instability regions for six-step Adam's methods.

We made some numerical experiments setting $\left|R_{1}(z)\right|=1$ with the aim to obtain the borders of the stability regions for Adam's methods.

Fig. 1 shows the stability regions (left sides of curves) for three-step Adam's methods $(N=2)$ with uniformly distributed nodes (1) and for nodes as zeroes 


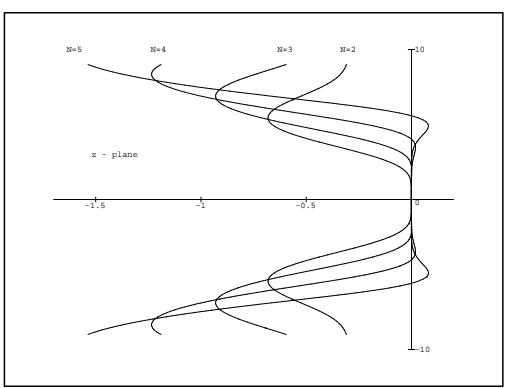

Figure 3. The borders of instability regions for methods with uniformly distributed nodes.

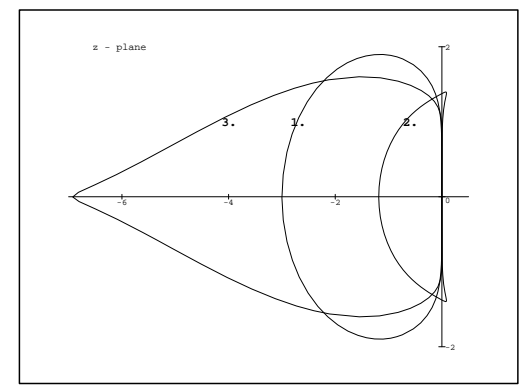

Figure 5. The optimization by sampling c for Adam's methods with uniformly distributed nodes.

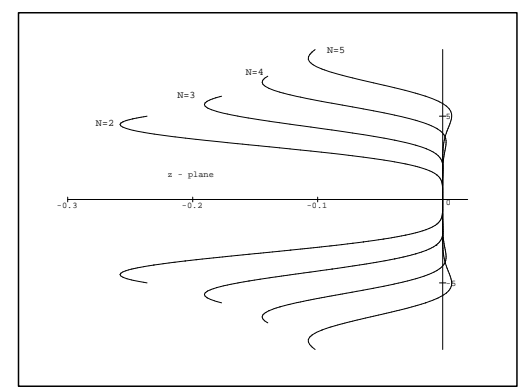

Figure 4. The borders of instability regions for methods with nodes as zeroes of Chebyshev polynomials of the second kind.

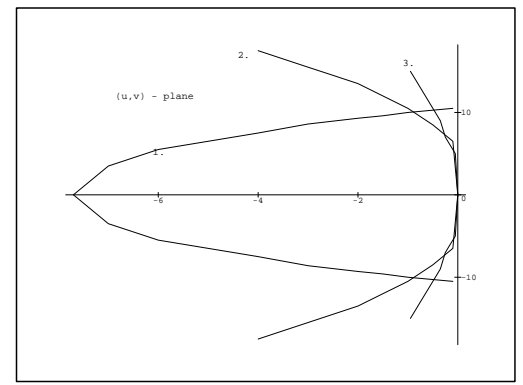

Figure 6. The borders of stability regions for six-step Adam's methods obtained by numerical experiments.

of the orthogonal polynomials: Jacobi (2), Chebyshev of the second kind (3), Legendre (4), Chebyshev of the first kind (5).

Fig.2 shows the corresponding results for six-step Adam's methods $(N=5)$.

Fig. 3 and Fig. 4 show the borders of stability regions for three $(N=2)$, four $(N=3)$, five $(N=4)$ and six $(N=5)$ step Adam's methods with uniformly distributed nodes and nodes as zeroes of Chebyshev polynomials of the second kind respectively.

We also made some experiments choosing the values of the constant $c$ with the aim to increase the stability domain of these methods.

Fig. 5 shows the borders of stability regions obtained by the root condition for characteristic equations of Adam's methods with uniformly distributed nodes in the cases: 1) $N=2, c=0$; 2) $N=4, c=0$; 3) $N=4, c=-0.017$.

In the Fig. 6 are shown the borders of stability regions obtained by numerical experiments for the following test problem $y_{1}^{\prime}=2 u y_{1}-\left(u^{2}+v^{2}\right) y_{2} ; y_{2}^{\prime}=y_{1}$; $y_{1}(0)=1 ; y_{2}(0)=0$ with two parameters $u$ and $v$. There are three curves: 
for six-step Adam's methods with uniformly distributed nodes (curve 1); with nodes as zeroes of Chebyshev polynomials of the second kind (curve 2), and of the first kind (curve 3 ).

\section{CONCLUSIONS}

- The inequality $\left|R_{1}(z)\right|<1$ with the first stability function $R_{1}(z)$ gives the stability domain for generalized Adam's methods (2.7) and (2.8) only for small enough $|z|$ or small enough length $H$ of the mold.

- A choice of the mold with nodes as zeroes of classical orthogonal polynomial for Adam's methods leads to schemes having the stability domain greater than one with the same number of uniformly distributed nodes.

- The special choice of constant $c$ in (2.7) and (2.8) can give both explicit and implicit schemes. If $c=0$ the method has order of one higher than order in the case when $c \neq 0$. However, the special choice of $c$ can also lead to the extension of the stability domain of methods.

- For Adam's methods the length of mold $H$, the step $h$ and the number of nodes $N+2$ in the mold are not independent. For example, $H, h$ and the number of Chebyshev nodes $x_{k}=-\cos (k \pi) /(N+1), k=0,1, \ldots, N+1$, in the one mold are connected by inequality $h \leq H \sin ^{2}(k \pi) /(2 N+2)$. Therefore, increasing of $N$ implies rapidly decreasing of step $h$ if the length of the mold $H$ is constant. Due to these reasons it is not possible by multistep Adam's methods to obtain the solution with an arbitrary precision, as was done by one step DM methods [1].

\section{REFERENCES}

[1] E. Haier, G. Wanner. Solving Ordinary Differential Equations Vol.1: Nonstiff Problems. Springer Verlag, Berlin, 1993.

[2] E. Haier, G. Wanner. Solving Ordinary Differential Equations Vol.2: Stiff and Differential-Algebraic Problems. Springer Verlag, Berlin, 1996.

[3] T. Cirulis, O. Lietuvietis. Degenerate Matrix Method for Solving Nonlinear Systems of Differential Equations. Mathematical Modelling and Analysis, 31998.

[4] T. Cirulis, D. Cirule, O. Lietuvietis. Multistep Degenerate Matrix Method for Ordinary Differential Equations. Mathematical Modelling and Analysis, 6 (1), 200158 - 67.

\section{Apibendrinto Adamso metodo analizè}

T. Cirulis, O. Lietuvietis

Darbe nagrinejjamas daugiažingsnis Adamso metodas. Jis formuluojamas kaip atskiras atvejis bendros išsigimstančiu matricu schemos. Naudojantis rezidiumų metodu ištirtas Adamso metodo stabilumas. Palygintos stabilumo sritys, kai diskretieji mazgai yra pasiskirstę tolygiai ir netolygiai. 